PROFESIONALES Y HERRAMIENTAS PARA EL DESARROLLO LOCAL Y SUS SINERGIAS TERRITORIALES. EVALUACIÓN Y PROPUESTAS DE FUTURO IX Coloquio Nacional de Desarrollo Local del GTDL-AGE 

ANTONIO MARTÍNEZ PUCHE, XAVIER AMAT MONTESINOS, ISABEL SANCHO CARBONELL y DANIEL SANCHIZ CASTAÑO (EDS.)

\section{PROFESIONALES Y HERRAMIENTAS PARA EL DESARROLLO LOCAL Y SUS SINERGIAS TERRITORIALES. EVALUACIÓN Y PROPUESTAS DE FUTURO}

IX Coloquio Nacional de Desarrollo Local del GTDL-AGE

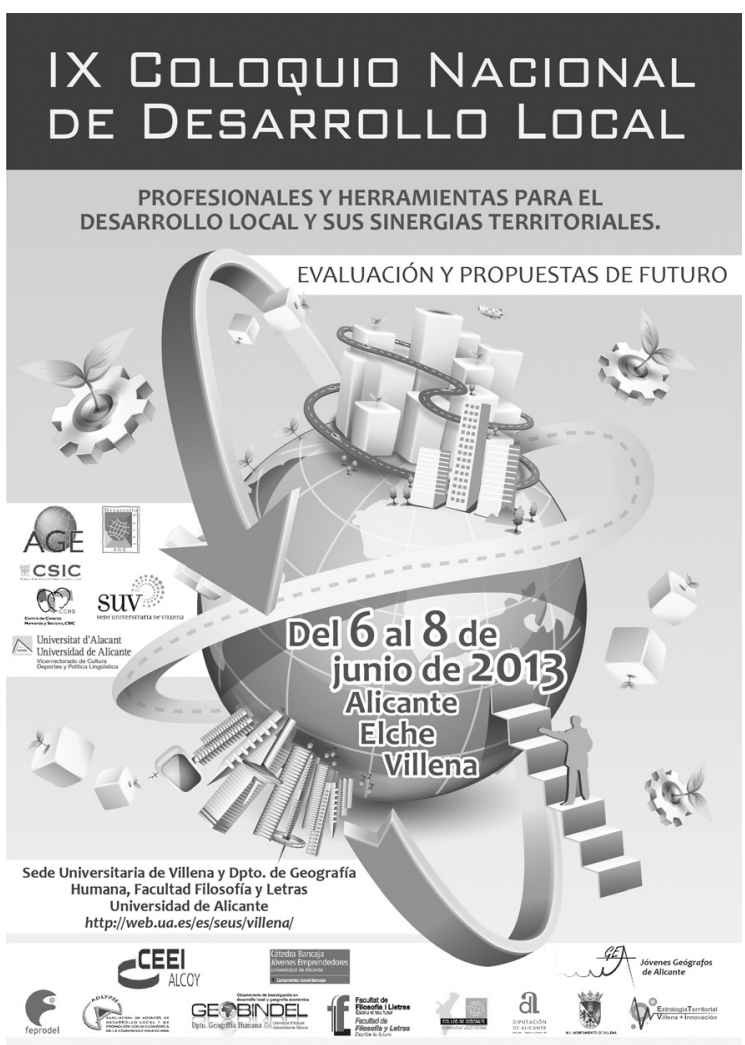


Este libro ha sido examinado y valorado por evaluadores ajenos a la Universidad de Alicante, con el fin de garantizar la calidad científica del mismo.

Publicacions de la Universitat d'Alacant

03690 Sant Vicent del Raspeig

Publicaciones@ua.es

http://publicaciones.ua.es

Telèfon: 965903480

(C) Antonio Martínez Puche, Xavier Amat Montesinos,

Isabel Sancho Carbonell y Daniel Sanchiz Castaño (eds.), 2016

(C) d'aquesta edició: Universitat d'Alacant

ISBN: 978-84-16724-00-0

Dipòsit legal: A 92-2016

Disseny de coberta: candela ink

Composició: Página Maestra (Miguel Ángel Sánchez Hernández)

Impressió i enquadernació: Guada Impresores

\section{unte \\ Unión de Editoriales
Universitarias Españolas \\ WWW.une.es
WWA}

Esta editorial es miembro de la UNE, cosa que garantiza la difusión y comercialización nacional y internacional de sus publicaciones.

Reservados todos los derechos. Cualquier forma de reproducción, distribución, comunicación pública o transformación de esta obra sólo puede ser realizada con la autorización de sus titulares, salvo excepción prevista por la ley. Diríjase a CEDRO (Centro Español de Derechos Repográficos, www.cedro.org) si necesita fotocopias o escanear algún fragmento de esta obra. 


\title{
SINERGIAS EMERGENTES EN EL ÁMBITO LOCAL COMO ALTERNATIVA PARA EL DESARROLLO EN LAS CIUDADES DE LA GRAN ÁREA METROPOLITANA DE COSTA RICA
}

\author{
David Porras Alfaro* \\ Departamento de Geografía - Universidad Autónoma de Madrid \\ david.porras@uam.es \\ Kenia García Baltodano* \\ Departamento de Geografía - Universidad Autónoma de Madrid \\ kenia.garcia@estudiante.uam.es
}

RESUMEN

El propósito de este estudio fue determinar sinergias entre actores y acciones para el desarrollo local. Mediante un estudio exploratorio se elaboró un inventario de actuaciones turísticas, culturales, de interacción social y mejoramiento urbano que buscan diversificar la economía de la Gran Área Metropolitana. Como resultado se identificaron 70 actuaciones con impacto en la última década. Se concluye que se ha experimentado una creciente atención a las temáticas vinculadas a la cultura y la ciudad, el fomento a la participación ciudadana y la identificación de nuevos tipos de turismo que pretenden impulsar el desarrollo local.

Palabras claves: desarrollo local, turismo, cultura, Gran Área Metropolitana, Costa Rica.

\section{RISING SYNERGIES IN THE LOCAL SPHERE AS AN OPTION TO DEVELOPMENT IN THE CITIES OF THE GRAN METROPOLITAN AREA OF COSTA RICA}

\footnotetext{
* Grupo de Investigación en Estudios Urbanos y del Turismo (URByTUR), oficialmente aprobado por la Universidad Autónoma de Madrid.
} 


\section{AbSTRACT}

This paper aims to determine synergies between social actors and actions for local development. By means of an exploratory research the authors created an inventory of projects related to tourism, culture, social interaction and urban improvements which intended to diversify the economy of the Gran Área Metropolitana. The outcome of this analysis was the identification of 70 projects which had a certain level of significance in the last decade. The authors came to the conclusion that the importance of topics such as culture and city, promotion of social participation and identification of new types of tourism oriented towards local development has considerably increased in the study area.

Key words: local development, tourism, culture, Gran Área Metropolitana, Costa Rica.

\section{CONSIDERACIONES INICIALES}

En las economías menos desarrolladas dependientes de los países con más poder adquisitivo y productivo, se presenta una mayor fragilidad socioeconómica en contextos de crisis, siendo un hecho obligado la búsqueda de alternativas que permitan salir de la inestabilidad. En el caso de Costa Rica - nación centroamericana con una superficie de $51.100 \mathrm{~km}^{2}$ y con una población de 4.301 .712 habitantes (Censo 2011) -según el Informe XVIII- Estado de la Nación (2012), el país ha presentado una reducción importante en su crecimiento y en la generación de empleo, a diferencia de otras naciones latinoamericanas.

La industria turística es uno de los principales sectores económicos, producto de los esfuerzos realizados en décadas recientes para promover a Costa Rica como un destino turístico sostenible y consolidar su imagen conservacionista. Los principales atractivos, hasta ahora explotados, han sido los recursos naturales y paisajísticos sobresaliendo el turismo de sol y playa, el de aventura y el ecoturismo, actualmente se puede decir que estos constituyen productos consolidados. Según el Estudio Económico de la GAM (CINPE-UNA, 2007) se suman también el turismo rural y el agroturismo conformando una masa importante dinamizadora de la economía que visita al país atraída por los extraordinarios atributos culturales y ambientales.

A pesar de ello, el rápido desarrollo turístico del resto de Centroamérica así como la necesidad de innovar para diversificar el sector, han provocado que se vuelque la mirada hacia incipientes nichos de mercado ligados al turismo urbano y cultural, además de las nuevas formas de consumo del espacio por parte de la ciudadanía y los visitantes. Lo que genera una hipotética base para el desarrollo de nuevos circuitos o la articulación con los itinerarios turísticos ya afianzados. 


\begin{abstract}
"(...) el turismo cultural tiene un perfil bajo, que se puede explicar por una especie de complejo de minusvalía, al considerarse que no existía nada sobresaliente en ese campo, la ausencia casi total de elementos coloniales que hacían lucir a nuestro país poco atractivo, comparado con la riqueza colonial de las demás naciones centroamericanas y, una inadecuada valorización de la riqueza de las diferentes manifestaciones regionales, cayéndose en unos pocos estereotipos que se reproducen incansablemente en los 'lobbies' de los hoteles y las ventas de artesanías." (CORRALES, 2006:6).
\end{abstract}

Las ciudades de la Gran Área Metropolitana (GAM) de Costa Rica (subregión más importante del país con un 3,44\% de su territorio, pero con el 52,71\% de la población), hasta ahora dedicadas principalmente a la residencia, el comercio y la industria han empezado a proyectarse como incipientes destinos turísticos, en especial sus cabeceras de provincia (Alajuela, Heredia, San José y Cartago) al ser las de mayor jerarquía dentro del sistema de ciudades (Figura 1). Si bien se trata de ciudades intermedias que junto a otros cantones forman la conurbación más importante del país, internamente se pueden distinguir características urbanas y culturales con identidad singular, que puestas en valor pueden favorecer la generación de iniciativas ligadas al desarrollo local a través del turismo, con un mercado interno en fase inicial.

Aparte de esto, la GAM reúne a lo interno un conjunto de elementos asociados a servicios, grandes empresas de alta tecnología y manufactura, así como otros espacios ligados al ocio y el tiempo libre (parques urbanos, restaurantes, cines, teatros, cafeterías, estadios, etc.) que la convierten en un lugar atractivo para la localización de personas y de actividades económicas. Asimismo, estas iniciativas buscan alejar el peligro de despoblamiento y abandono que sufren los principales centros históricos.

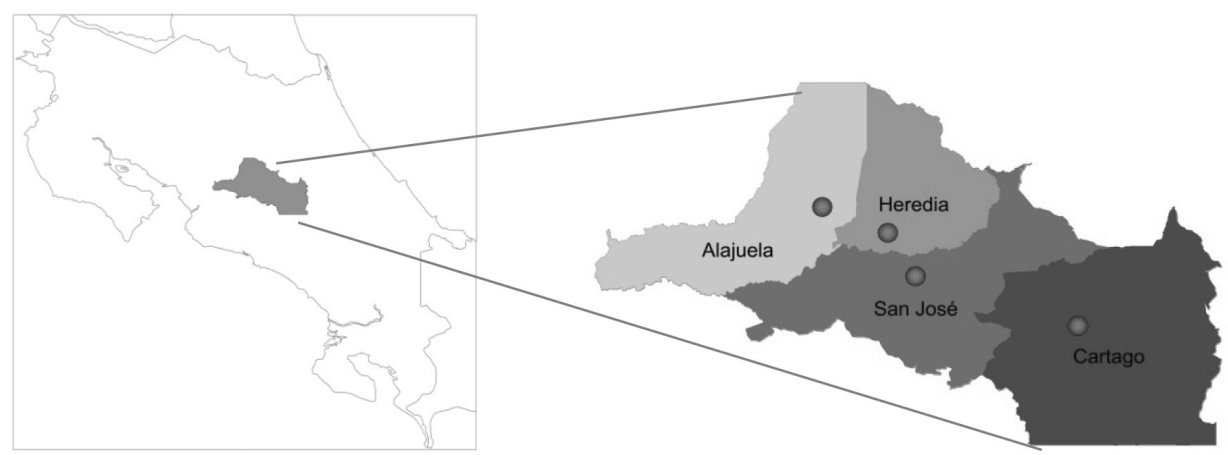

Figura 1. Ámbito de estudio (Gran Área Metropolitana de Costa Rica).

Fuente: elaboración propia. 
En este sentido, en la última década llama la atención el surgimiento de un conjunto de acciones vinculadas a la cultura, el turismo y el mejoramiento urbano planteadas desde las instituciones de Gobierno, la cooperación internacional, los ayuntamientos, el sector público y las organizaciones no gubernamentales o las iniciativas ciudadanas.

Con la participación ciudadana como motor fundamental, sus efectos positivos empiezan a notarse en la rehabilitación de inmuebles de interés histórico arquitectónico y zonas urbanas degradadas, el fomento de la animación sociocultural, la construcción de equipamiento, entre otras acciones que en conjunto repercuten indirectamente en un mayor aprovechamiento de la ciudad y la generación de capital. Particularmente los aspectos vinculados al arte y la cultura comienzan a ser redescubiertos y a tomar un protagonismo novedoso en Costa Rica; todas estas operaciones han favorecido el interés de los gobiernos locales por explotar los recursos urbanos y culturales dentro de su oferta, en especial en sus cascos históricos.

Si bien se trata de actuaciones realizadas sin una coordinación intermunicipal, en la mayoría de los casos y con presupuestos limitados, la proximidad existente entre estas ciudades hace pensar en la posibilidad de establecer sinergias entre los diversos actores y plantear una estructura organizativa que permita un mejor vínculo e interacción entre ellas. Por tanto, esta aportación plantea generar como principal resultado un inventario de las distintas actuaciones que evidencian estas dinámicas, para establecer un estado de la cuestión que favorezca el engranaje de las acciones individuales existentes y permita expandir la promoción de alternativas factibles para el desarrollo local, desde una óptica novedosa en el país.

\section{Aportes para el Desarrollo local}

Desde esta aportación se concibe el desarrollo local integralmente como una opción válida para la obtención de resultados reales en el territorio, en momentos de recesión económica mundial y necesidad de un mejoramiento en la calidad de vida de la población. El aprovechamiento de la dimensión territorial, con la heterogeneidad y complejidad de cada región, busca identificar sus particularidades biofísicas, sociales, culturales, ambientales y otras acciones de tipo ciudadanas y empresariales (en todas su escalas). Producto de la incorporación de agentes públicos y privados, en conjunto con la sociedad civil, se debe establecer una ruta para resolver los principales desafíos en materia de medio ambiente, desarrollo humano y económico.

La cultura debe tener un papel protagónico en las estrategias de desarrollo, en los programas y proyectos para lograr un acercamiento de la población y las instituciones con un enfoque compartido. "(...) en definitiva, como un factor de enriquecimiento personal, de cohesión social, de identidad colectiva, de 
regeneración urbana, de proyecto exterior, de desarrollo económico y así mismo de mejora de la calidad de vida del territorio". (POSE, 2006:25). Se debe apuntar hacia la consecución de objetivos vinculados a la generación de capital social, incrementos productivos y el mejoramiento del sentido de pertenencia y participación ciudadana.

Ávila y Barrado (2005), en relación a las nuevas tendencias turísticas de aprovechamiento local, mencionan la posibilidad de que el turismo se entienda como una actividad compleja y participativa. Con ello se empiezan a desarrollar ámbitos que hasta el momento se concebían como marginales o apartados totalmente de los circuitos tradicionales de este sector productivo, como el caso de la GAM con ciudades cabeceras de provincia que servían simplemente de paso. De ahí la importancia de implementar políticas, estrategias de diseño, promoción y comercialización planificadas por medio de consensos sociales y una implicación de todos los sectores que en conjunto constituyen la población de acogida del destino. El destino turístico requerirá, además de una planificación sectorial, una correcta ordenación territorial con el fin de considerar de forma conjunta los aspectos ligados a la producción junto con otras de carácter espacial (Barrado, 2004).

Pensar en un desarrollo local a través de la generación de iniciativas ligadas al turismo, la cultura y el mejoramiento urbano no es algo novedoso, sin embargo, en una subregión como la GAM estos aspectos no han tenido en décadas recientes el mejor abordaje. Pese a ello, y como se constatará más adelante, la situación en los últimos años empieza a cambiar producto en algunos casos de impulsos estatales, municipales, la cooperación internacional o las propias iniciativas ciudadanas, quizás estas últimas las que más han insistido en la reactivación del tema por la necesidad de obtener nuevas opciones para el desarrollo y la creciente preocupación por la pérdida del espacio urbano.

Un trabajo sinérgico de este tipo a largo plazo mejora la competitividad, impulsa el desarrollo de obra pública y privada, el clima de inversión, la generación de empleo de calidad, la identificación de los ciudadanos con su entorno y el impulso de un desarrollo equitativo de las diferentes regiones del país. Sin perder de vista, que todos estos esfuerzos, si realmente se quiere que tengan una incidencia constante sobre el desarrollo local, requerirán de una consolidación en el tiempo, incentivos, construcción de servicios urbanos (físicos y virtuales); y en el caso del turismo urbano y de interior, un aumento cuantitativo de la demanda y su fortalecimiento en cuanto a permanencia y capacidad de gasto (Valenzuela, 2008).

\section{HiPÓTESIS Y METODOLOGÍA}

Como hipótesis del estudio se plantea que las ciudades de la GAM muestran un aumento significativo de acciones vinculadas al turismo y la cultura, hecho 
que a través de las sinergias producto de la participación de la población, impulsan el desarrollo local, el mejoramiento de la calidad urbana y los procesos de apropiación del espacio.

Metodológicamente para el abordaje de este estudio se realizó un inventario exploratorio que reúne las principales iniciativas llevadas a cabo en las cuatro ciudades cabeceras de provincia de la GAM, así como algunas de mayor envergadura que aunque trascienden los límites municipales de dichas ciudades, fueron tomadas en consideración por el impacto que tienen para la subregión.

Este inventariado se basa en un levantamiento de información elaborado a partir de las diferentes fuentes de difusión oficiales de las propias entidades, entre ellas páginas web, redes sociales, blogs de los diferentes actores (entes estatales, municipalidades, oficinas de turismo, organismos no gubernamentales) que intervienen en los procesos, así como información publicada en prensa y consulta directa a los actores participantes.

Debe aclarase que este proceso de inventariado y aproximación a la temática no persigue en ningún caso una pretensión de exhaustividad pues superaría por mucho los objetivos y posibilidades de este trabajo, tan sólo se ha querido ejemplificar y obtener conclusiones preliminares que requerirán de un trabajo con otras condiciones (realización de entrevistas, visitas de campo, etc.) para llegar a una mayor profundización en los resultados.

\section{ANÁlisis DE RESUltados}

La realización del inventario (Tabla 1) permitió analizar una serie de aspectos en relación con las actuaciones que los diversos agentes llevan a cabo en el territorio y cómo estas se vinculan a la búsqueda de alternativas al desarrollo. Del mismo modo, se ha tratado de caracterizar cada una de las ciudades analizadas a través del perfil que brindan las intervenciones y el sector que lo promueve.

Como se muestra en el Gráfico 1 las principales actuaciones estudiadas corresponden a los equipamientos, en la mayoría de los casos de tipo cultural. En segundo lugar se encuentran las intervenciones urbanas, que abarcan dos clases: el espacio urbano y el planteamiento de nuevos proyectos de gran escala y trascendencia para las ciudades (elegidos recientemente mediante concursos abiertos de diseño dirigidos a profesionales del sector).

La promoción de la cultura (que incluye la recuperación y puesta en valor del patrimonio, la difusión de las artes y un creciente apoyo al sector artístico) planteada desde los gobiernos locales y el Estado, así como un número destacable de actividades de animación sociocultural ideadas y ejecutadas tanto, por el sector privado como el público, paulatinamente se convierten en catalizadores de un mayor vínculo social y de la generación de nuevos atractivos de las ciudades. La participación ciudadana en este proceso ha sido vital, pues la ciudadanía no sólo ha tenido un rol de consumidor, sino que muchos colectivos, 


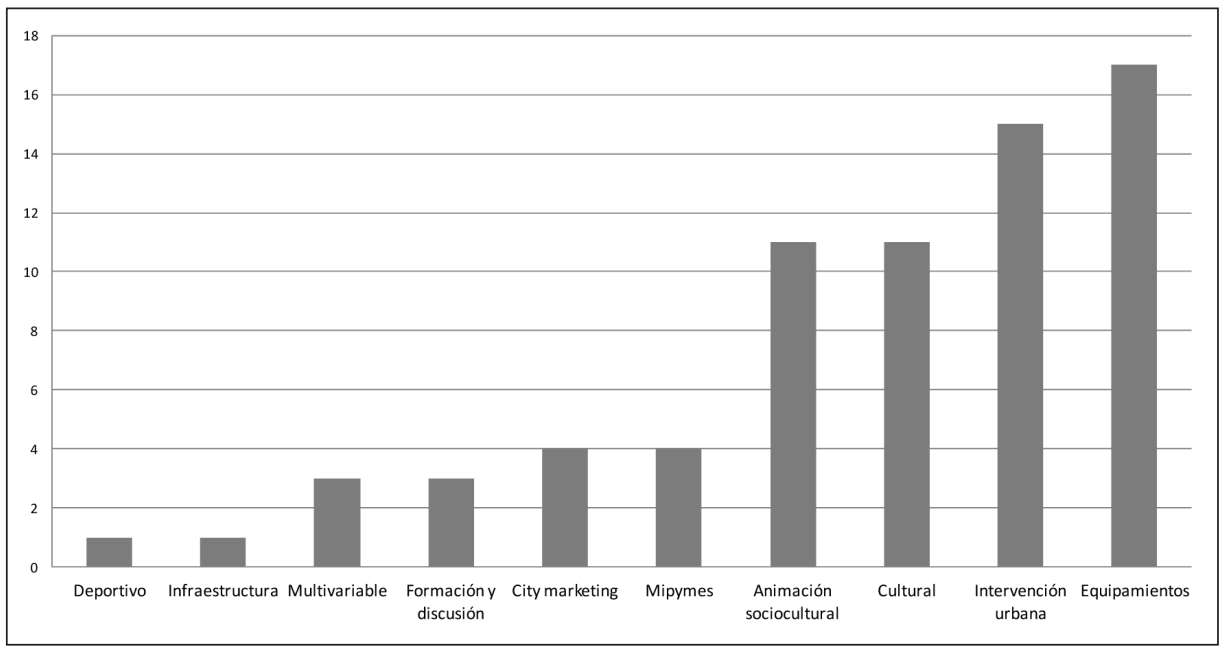

Gráfico 1. Categorías según tipo de actuación.

Fuente: elaboración propia.

organizaciones no gubernamentales y empresas plantean actuaciones concretas. Estas por una parte contribuyen a la apropiación ciudadana del espacio y por otro lado, generan actividades que promueven el desarrollo social y cultural, algunos ejemplos claros son los recorridos urbano-patrimoniales en cada una de las ciudades y actividades como el Art City Tour y 100 en un día en la ciudad de San José (Tabla 1).

Un aspecto a destacar es que tres de las cuatro ciudades analizadas (San José, Alajuela y Cartago) han iniciado procesos ligados al desarrollo de productos turísticos a través de la creación de oficinas de turismo, sitios web y estrategias incipientes de city marketing, si bien estas necesitan de una mayor potenciación son herramientas orientadas a conquistar nuevos visitantes. A esto se suma la realización de grandes eventos nacionales e internacionales (festivales, ferias, exposiciones, certámenes) que permiten demostrar las capacidades en términos de equipamiento y capital humano que ofrecen estas ciudades, así como sus atractivos urbanos y culturales.

Algunas grandes iniciativas que se están implementando tienen un carácter multivariable pues incluyen una serie de factores como la dotación de equipamientos, la formación de capital humano, el apoyo a las micro, pequeñas y medianas empresas (mypimes), el desarrollo sociocultural, entre otros.

Un elemento a resaltar es la posibilidad de potenciar los vínculos sinérgicos entre las ciudades de la GAM a través de la recuperación del servicio de tren. Luego de un período de inactividad de más de una década, se ha retomado este medio de transporte para la realización de viajes urbanos e interurbanos de 
pasajeros. En la actualidad el tren comunica a Heredia y Cartago con San José y se espera que para 2014 se amplíe el servicio a Alajuela, constituyéndose en una alternativa más sostenible para la comunicación de las ciudades.

En referencia a la situación particular de cada ciudad, según los datos obtenidos, Alajuela muestra un notable trabajo de cooperación entre el sector público y privado, que ha dado como resultado una serie de actuaciones de impacto urbano en un corto tiempo respecto a las demás provincias. El mejoramiento de una parte significativa de su espacio público y la recuperación de importantes edificaciones patrimoniales propician una imagen más atractiva para propios y visitantes. No deja de destacar la ausencia de iniciativas únicamente privadas en la ciudad.

Al igual que en el caso anterior, Cartago muestra una notoria colaboración público-privada para el desarrollo de las actuaciones. Destaca el involucramiento de la ciudadanía en la programación de actividades de animación sociocultural y el mejoramiento de la calidad urbana a través de intervenciones, a saber la nueva ciclovía y la dotación de equipamientos como el Museo y el Anfiteatro Municipal (Tabla 1).

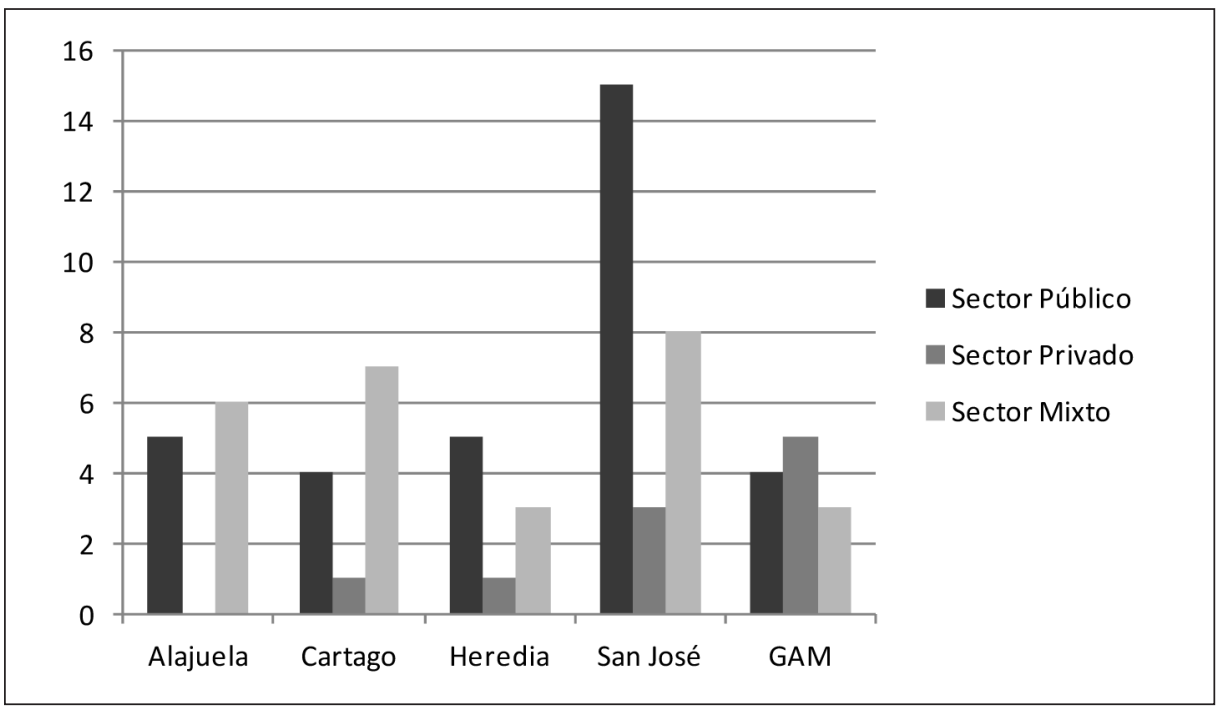

Gráfico 2. Categorías según el sector promotor.

Fuente: elaboración propia.

Respecto a San José, como capital del país, la inversión pública (municipal y estatal) es mucho más notoria que en el resto de zonas analizadas. Las actuaciones que se han realizado han sido orientadas a revitalizar la ciudad y atraer nuevas inversiones, aumentando con ello significativamente su competitividad territorial. Grandes eventos de carácter deportivo y cultural como los Juegos 
Deportivos Centroamericanos y el Festival Internacional de las Artes (Tabla 1) se constituyen en importantes vitrinas para mostrar los atributos josefinos; además del impulso que supone la recuperación de espacios urbanos y construcción de nuevo equipamiento para tales fines.

Por su parte, la ciudad de Heredia presenta el menor número de actuaciones inventariadas, las cuales se orientan principalmente a promocionar la cultura a través de la mejora de equipamientos, la realización de actividades y el favorecimiento a grupos de artistas y artesanos (capacitaciones y facilidades para realizar exposiciones de sus obras). Sumado a esto, el trabajo realizado en cuanto al remozamiento del patrimonio edificado de la ciudad y la intervención del espacio urbano ha sido importante para el mejoramiento de su imagen urbana. Las acciones se realizan principalmente desde el sector público.

En relación a las actuaciones puntuales dentro de la GAM, se han destacado algunos ejemplos planteados desde los ámbitos público, privado y mixto como casos de gran impacto para la subregión que contribuyen desde diferentes áreas al desarrollo local.

Finalmente, debe mencionarse como aspecto destacable, el trabajo de extensión universitaria que realizan las instituciones de educación superior públicas (Universidad de Costa Rica, Universidad Nacional de Costa Rica, Universidad Técnica Nacional y el Instituto Tecnológico de Costa Rica) tanto en proyectos de interés nacional como en su entorno inmediato dentro de las ciudades de San José, Heredia, Alajuela y Cartago. Este apoyo resulta ser un gran impulso para estas comunidades y un enlace efectivo entre la población y la academia.

5. Inventario de actuaciones locales en las ciudades de la GAM (Tabla 1) 


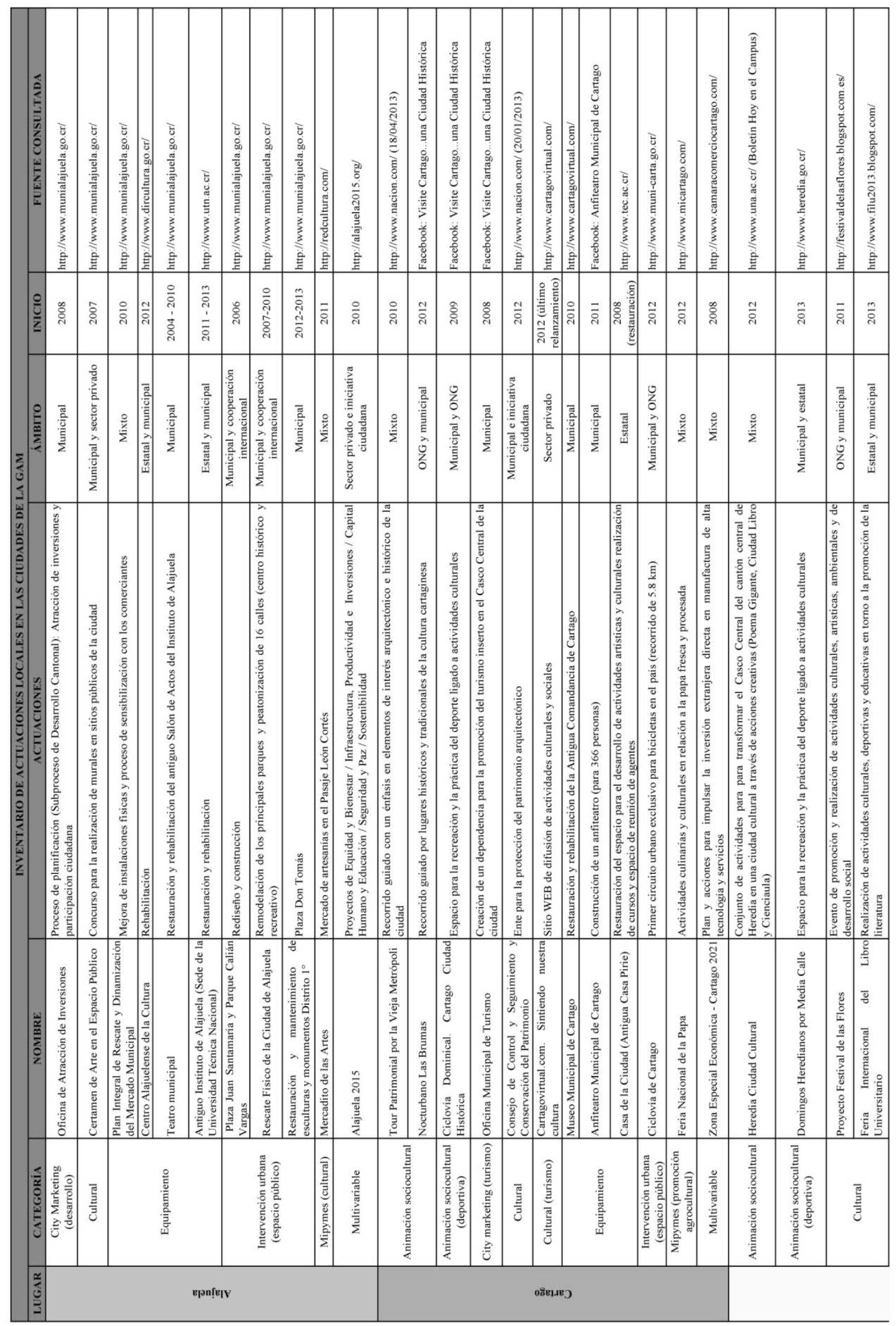




\begin{tabular}{|c|c|c|c|c|c|c|c|c|c|c|c|c|c|c|c|c|c|c|c|}
\hline 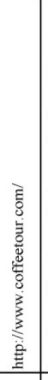 & 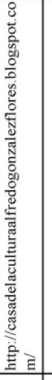 & 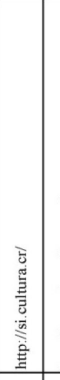 & 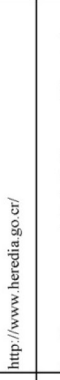 & 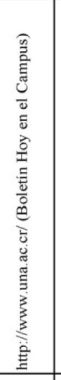 & 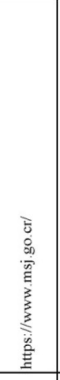 & 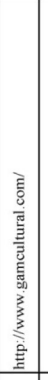 & 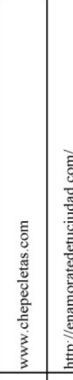 & 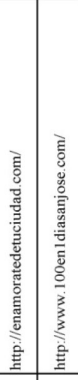 & 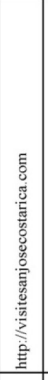 & 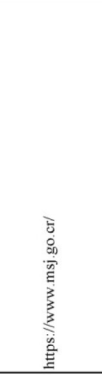 & 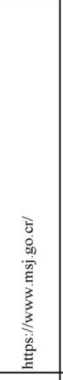 & 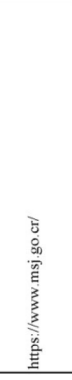 & 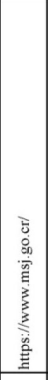 & 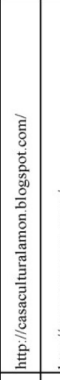 & 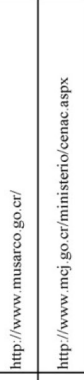 & 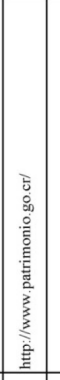 & 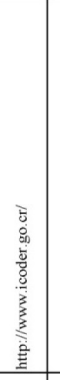 & 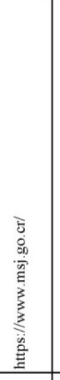 & 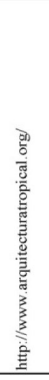 \\
\hline ब更 & 舷 & ปั & & ఫ్తి & $\stackrel{m}{\bar{c}}$ & ఫ्रे| & \begin{tabular}{l|l}
\multirow{2}{*}{} & $\bar{a}$
\end{tabular} & 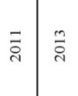 & 永 & \&్ & : & : & \begin{tabular}{l|} 
\\
0 \\
0
\end{tabular} & $\begin{array}{l}\infty \\
\vdots \\
\vdots\end{array}$ & 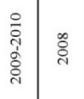 & 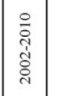 & $\begin{array}{l}\overline{\bar{̆}} \\
\text { \$े }\end{array}$ & 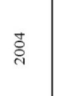 & 总 \\
\hline 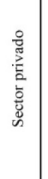 & 焉 & 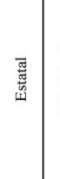 & 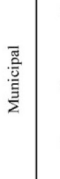 & 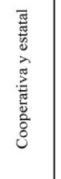 & 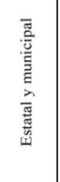 & 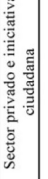 & 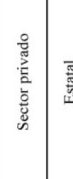 & 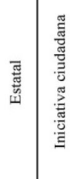 & $\mid \begin{array}{l}\text { 営 } \\
\frac{\bar{g}_{\overline{2}}}{2}\end{array}$ & 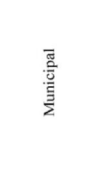 & $\begin{array}{l}\frac{\bar{a}}{2} \\
\frac{0}{20} \\
\frac{\bar{z}}{2}\end{array}$ & $\begin{array}{l}\frac{\sqrt{3}}{20} \\
\frac{a}{2}\end{array}$ & 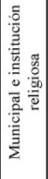 & 焉 & 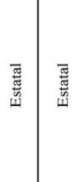 & 喜 & 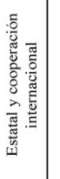 & 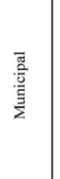 & 辯 \\
\hline 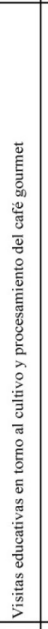 & 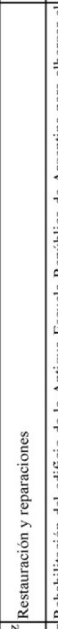 & 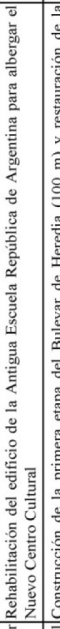 & 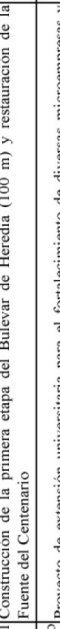 & 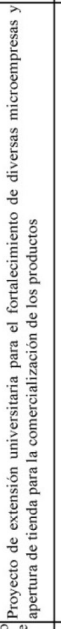 & 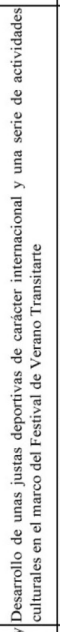 & 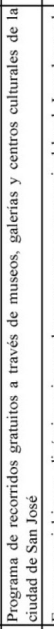 & 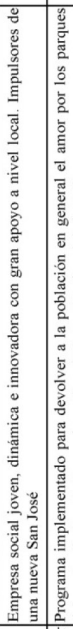 & 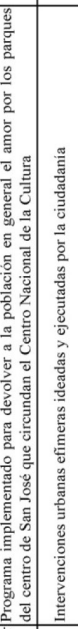 & 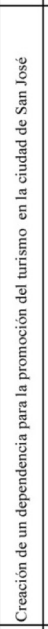 & 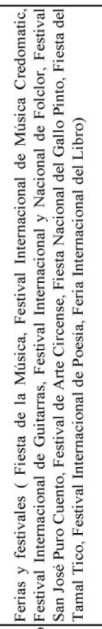 & 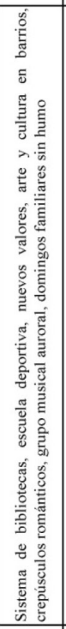 & 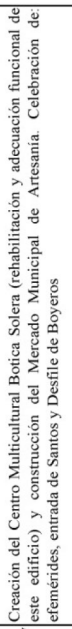 & 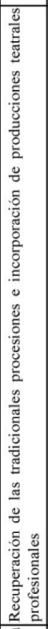 & 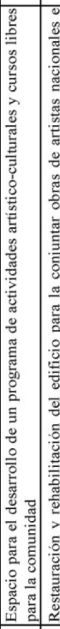 & 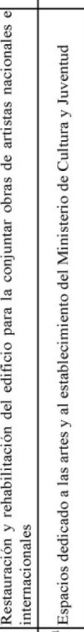 & 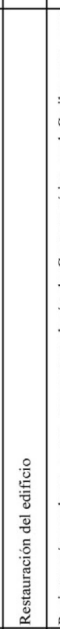 & 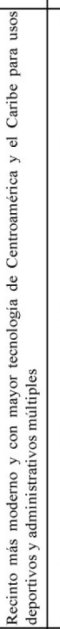 & 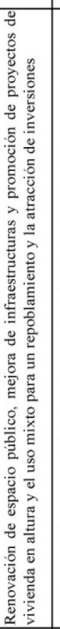 & 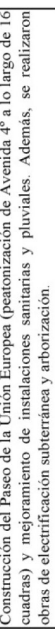 \\
\hline 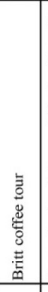 & 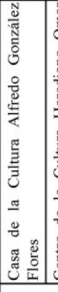 & $\begin{array}{l}\frac{2}{3} \\
\frac{\pi}{3} \\
\frac{\pi}{2}\end{array}$ & 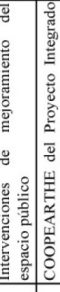 & 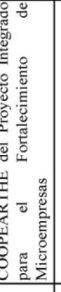 & 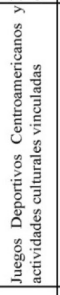 & 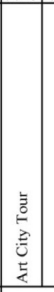 & 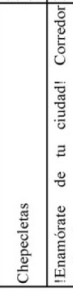 & 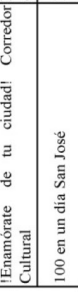 & 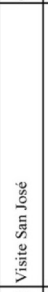 & 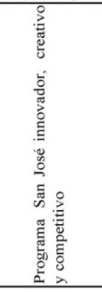 & 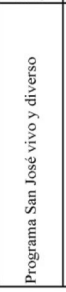 & 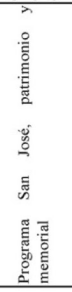 & 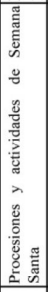 & 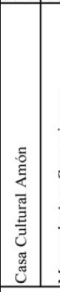 & 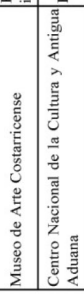 & 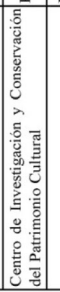 & 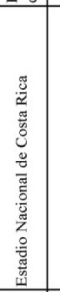 & 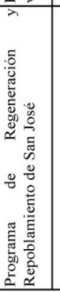 & 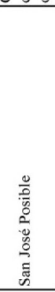 \\
\hline 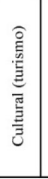 & & & 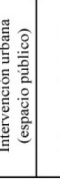 & 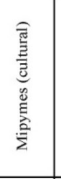 & $\begin{array}{l}\text { 咅 } \\
\text { do }\end{array}$ & & & & 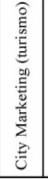 & & है & & 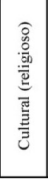 & & & & & & \\
\hline \multicolumn{5}{|c|}{ в!раююн } & \multicolumn{15}{|c|}{ gsof urs } \\
\hline
\end{tabular}




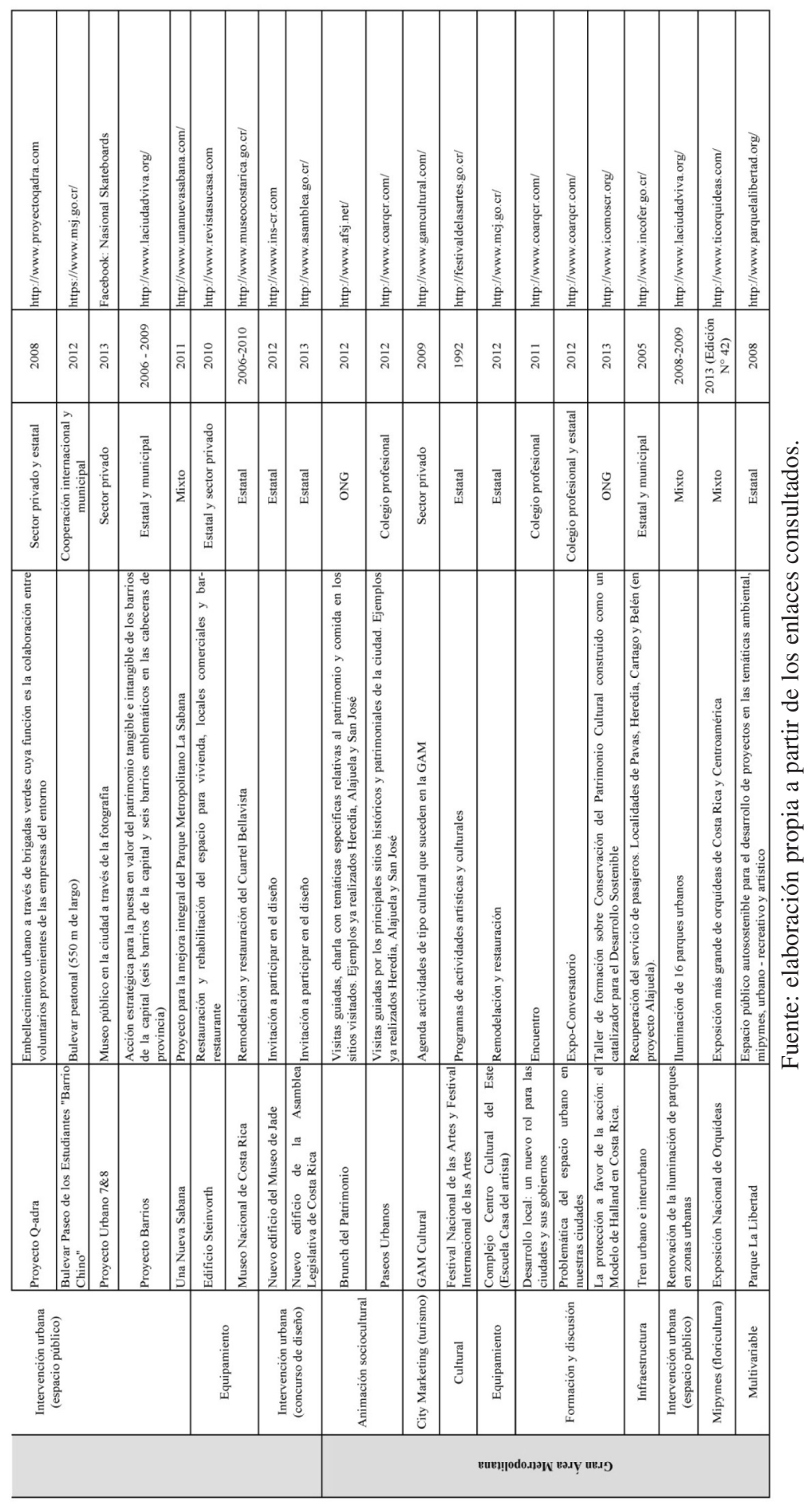




\section{CONCLUSIONES Y RECOMENDACIONES}

Con el inventario y análisis de resultados efectuados como parte de este estudio, se concluye que las cuatro ciudades cabeceras de provincia han experimentado, en la última década, una creciente atención a las temáticas vinculadas a la cultura y la ciudad, el fomento a la participación ciudadana y la identificación de nuevos tipos de turismo que pretenden impulsar el desarrollo local. Además estas iniciativas buscan contrarrestar los efectos negativos externos (crisis monetaria internacional y dependencia de la inversión extranjera) e internos (desigualdades en la distribución de la riqueza y un único nicho de mercado dirigido principalmente al turismo de sol y playa y naturaleza).

La identificación de estas actuaciones lleva a pensar en la necesidad de concretar mecanismos de sostenibilidad de dichas propuestas. Todas demandan un importante componente humano, el cual merece estar apoyado desde las instancias gubernamentales y municipales, favoreciendo la eclosión de nuevas sinergias.

Por otro parte se ha encontrado dificultad en el acceso a información sistematizada en torno a temas relacionados al turismo, la cultura y el desarrollo local, principalmente a nivel municipal. No obstante se destaca la labor del Ministerio de Cultura y Juventud en la elaboración de los primeros instrumentos en esta materia (SICultura y el Atlas de Infraestructura Cultural y Patrimonio de Costa Rica), así como del Programa Estado de la Nación, que incorporó en su edición más reciente una actualización de dicha temática a través del Capítulo 6. Producción cultural en Costa Rica: nuevos aportes para su conocimiento.

Sin lugar a dudas la implementación de tecnologías de la información y comunicación (TIC) han facilitado la creación de sinergias, sirviendo de enlace entre diferentes agentes y como mecanismo de difusión ante el resto de la población. Destaca la labor de los municipios en la construcción de portales web y la difusión del quehacer municipal a través de las redes sociales.

Se recomienda promover por parte del Instituto Costarricense de Turismo un balance en el contenido de las campañas turísticas nacionales y medios de difusión (ferias nacionales e internacionales, prensa, TIC) en las cuales se incentive una mayor vinculación a los recursos culturales y no simplemente a los centrados en aspectos naturales.

Finalmente, como posibles destinos turísticos es necesario que las ciudades lleven a cabo un proceso de mejoramiento en dos líneas, una enfocada a la creación de facilidades y servicios para el turista, y otro directamente vinculado a la población residente como usuario permanente del espacio. Las políticas a escala nacional y local requieren de un análisis integral del territorio, para ello los principales estudios y planes deben contar con una visión holística que contemple las sinergias con otros territorios, las relaciones sistémicas internas y las iniciativas que surgen desde la propia participación ciudadana. Sin la gestión y el planeamiento de estas no se podrán obtener resultados en el desarrollo local a largo plazo. 
BIBLIOGRAFÍA

ÁVILA, R. y BARRADO, D. (2005): "Nuevas tendencias en el desarrollo de destinos turísticos: marcos conceptuales y operativos para su planificación y gestión”, Cuadernos de turismo, ${ }^{\circ} 15$, p. 27-44.

BARRADO, D. (2004): "El concepto de destino turístico. Una aproximación geográfico-territorial", Estudios Turísticos, n 160, p. 45-68.

CASTELLANOS, A. (2012): Atlas de infraestructura y patrimonio cultural de las Américas: Costa Rica, México, Fundación Interamericana de Cultura y Desarrollo y Ministerio de Cultura y Juventud, Gobierno de Costa Rica, $192 \mathrm{p}$.

CENTRO INTERNACIONAL DE POLÍTICA ECONÓMICA PARA EL DESARROLLO SOSTENIBLE, UNIVERSIDAD NACIONAL DE COSTA RICA. (2007): Estudio Económico de la GAM (Estructura Económica), Heredia, Universidad Nacional de Costa Rica, $111 \mathrm{p}$.

CORRALES, F. (2006): "Turismo y cultura en Costa Rica", Tecnitur, n 118, p. 6.

INSTITUTO NACIONAL DE ESTADÍSTICA Y CENSO: Censo 2011, Instituto Nacional de Estadística y Censo, Gobierno de Costa Rica. http://www. inec.go.cr/, [consulta: 28/05/2013].

MINISTERIO DE CULTURA Y JUVENTUD: Sistema de Información Cultural, Ministerio de Cultura y Juventud, Gobierno de Costa Rica. http:// si.cultura.cr/, [consulta: 28/05/2013].

POSE, H. (2006): La cultura en las ciudades. Un quehacer cívico-social. Barcelona, $215 \mathrm{p}$.

PROYECTO ESTADO DE LA NACIÓN. (2012): Estado de la Nación en Desarrollo Humano Sostenible: Decimoctavo Informe de la Nación 2011, San José, Costa Rica, Proyecto Estado de la Nación, 436 p.

RAUSELL, P. (2007): Cultura. Estrategia para el desarrollo local, Madrid, Dirección General de Relaciones Culturales y Científicas-AECI, 289 p.

VALENZUELA, M. (2008): "Nuevos turismos para nuevos turistas. De la identificación a la captación de la clientela", en CEBRÍAN, F. (coord.), Turismo rural y desarrollo local, Cuenca, Universidad de Castilla-La Mancha: Secretariado de Publicaciones de la Universidad de Sevilla, p. 57-65. 